\title{
LOADING ON PIPELINES DUE TO EXTREME HYDRODYNAMIC CONDITIONS
}

\author{
Behnaz Ghodoosipour, University of Ottawa, bghod068@uottawa.ca \\ Jacob Stolle, University of Ottawa, jstol065@uottawa.ca \\ Ioan Nistor, University of Ottawa, inistor@uottawa.ca \\ Majid Mohammadian, University of Ottawa, majid.mohammadian@uottawa.ca \\ Adrian Raul Simpalean, University of Ottawa, asimp051@uottawa.ca
}

\section{INTRODUCTION}

Proper design of pipelines used for oil, gas, water and wastewater transmission is of great importance. This is even more critical when pipelines are located in nearshore, coastal areas that are exposed to extreme hydrodynamic events, such as tsunami and storm surges. The American Society of Civil Engineers (ASCE)), in its ASCE7 Chapter 6: Tsunami Loads and Effects, the new standard for tsunami impacts and loading stresses the necessity to study tsunami loads on pipelines. Understanding the hydrodynamic forces acting on the pipelines is vital in ensuring their safe operation and avoiding potential damage to the environment. To address these issues, the following study is the first of its kind to investigate loading on pipelines due to tsunami-like bores. Guidelines for Design of Structures for Vertical Evacuation from Tsunamis FEMA P646 define the drag force acting on a structure as:

$$
F_{d}=\frac{1}{2} \rho_{s} C_{d} B\left(h u^{2}\right) \max
$$

Where $\rho_{s}$ is the fluid density, $C_{d}$, drag coefficient, B width of the structure in the plane normal to the direction of flow, h, flow depth and $\mathrm{u}$, maximum flow velocity at the location of the structure.

\section{OBJECTIVES AND NOVELTY}

This study focused on measuring and analyzing the forces induced by extreme hydrodynamic events on submerged and above-ground pipelines. This was achieved by combining a comprehensive experimental study and detailed CFD numerical simulations. The study aimed to provide guidelines for the design of pipelines in tsunami-prone areas. At the same time, this study proposes specific values for drag coefficients in the case of a transient tsunami-like coastal flow for different relative gap ratios and submergence conditions.

\section{EXPERIMENTAL SETUP}

The experiments were conducted in the hydraulic Flume of the Hydraulics Laboratory at the University of Ottawa (Canada). The concrete flume is $30.1 \mathrm{~m}$ in length, $1.5 \mathrm{~m}$ in width, and $0.5 \mathrm{~m}$ in height. A swing gate was installed $21.55 \mathrm{~m}$ downstream from flume inlet and dam break waves with different initial impoundment depths were generated by the rapid opening of a swing gate. The steel pipe $(D=10 \mathrm{~cm}$ dia.) model was located $5.6 \mathrm{~m}$ downstream of the gate at different gap sizes (e, distance from flume bed). (Figures $1(a)$ and (b)). Experiments were conducted by varying the relative gap ratios $(e / D=0.3,0.6,0.8)$, still water depths for wet bed condition $(\mathrm{d}=0-8 \mathrm{~cm})$ and initial impoundment depths $(\mathrm{h}=30,40$ and $50 \mathrm{~cm})$. The components of the force exerted on the pipe were measured using a dynamometer connected to the pipe.

\section{NUMERICAL MODEL}

Experimental results were compared with the results of a new model developed using the OpenFOAM CFD Modeling tool. The model uses the Finite Volume Method to solve the 3-D Navier-Stokes equations. The model was used to simulate dam break flow impacting a pipe replicating the same conditions of the aboveexplained experimental setup.
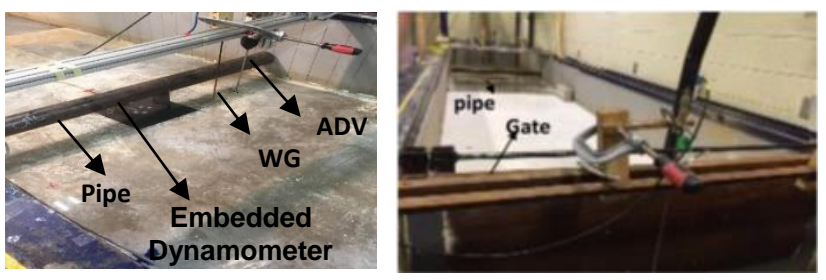

Figure 1 - a) Pipe location inside the flume, ADV and wave gauge, b) downstream view - flume and gate.

\section{RESULTS AND DISCUSSION}

Results for the force-time history for dry bed conditions and different gap ratios are shown in Figure 2(a) and (b). One can notice that, for e/D=0.3, the drag and lift force behavior are considerably different when compared to data obtained using other e/D ratios. This difference is due to the vortex shedding suppression behind the pipe as a results of the small e/D. This deflects a portion of the fluid from bottom upstream region of the flume over the top of the circular cylinder and thus reduced the flow passing through the gap. This caused a considerable impulse drag force, as well as a significant downward peak in lift force time history at bore impact time.

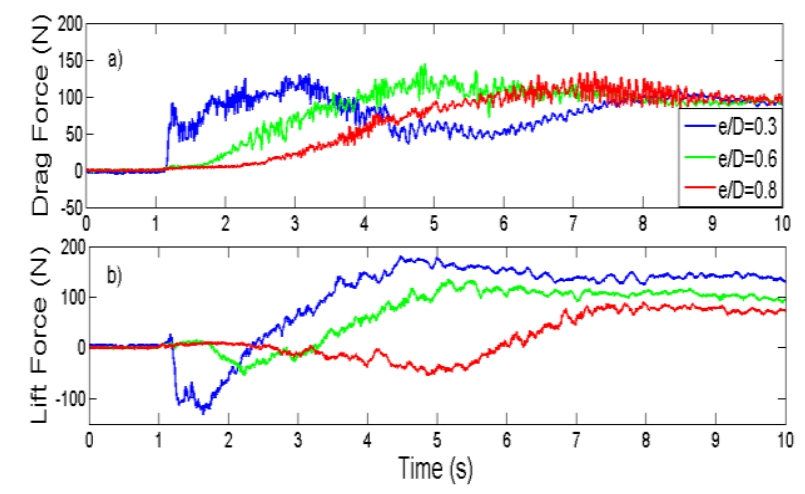

Figure 2 - Time-history of the a) drag force $b$ ) lift force for drybed condition, $\mathrm{h}=40 \mathrm{~cm}, \mathrm{e} / \mathrm{D}=0.3,0.6,0.8$.

Results from wet bed condition experiments, showed little sensitivity to changing e/D ratio. However, changing still water to impoundment depth ratio $(\mathrm{d} / \mathrm{h})$ showed to significantly affect the force time history both in horizontal and vertical directions. Results indicate that for $d / h$ ratios resulting in smaller initial level of pipe submergence, duration of the horizontal run-up force is considerably larger. Lift force showed a noticeable decrease by increasing $d / h$. 
A good agreement between numerical model results and experimental measurements were achieved. Results indicated that the Reynolds Averaged Navier Stokes (RANS) model using realizable k-epsilon turbulence model performed best when compared to the Large Eddy Simulation (LES) model. Figure 3 illustrates examples from the numerical model in dry and wet bed condition at $\mathrm{t}=0$ and at the time of bore impact. It was noticed that in wet bed condition, water level rises more significantly at the time bore impact compared to dry bed condition.

\section{Dry bed conditions}
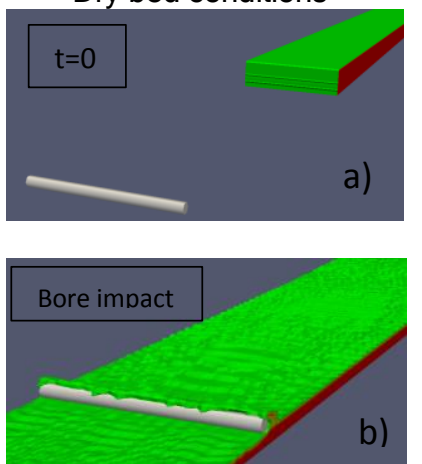

Wet bed condition
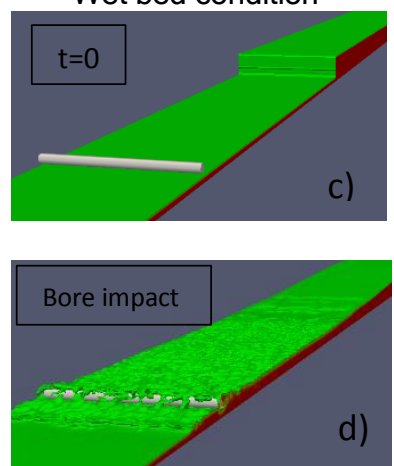

Figure 3 - 3D view of OpenFoam CFD model, $a$ \& b) Dry bed condition. $c \&$ d) Wet bed condition.

Figure 4 shows the comparison between results from realizable k-epsilon model and the experimental data for dry bed condition and $e / D=0.6$. The calculated coefficient of determinations were $R^{2}=0.8$ and $R^{2}=0.6$ for drag force and lift force respectively. The high $R^{2}$ values achieved, indicate acceptable fitting of observed and model estimated data.

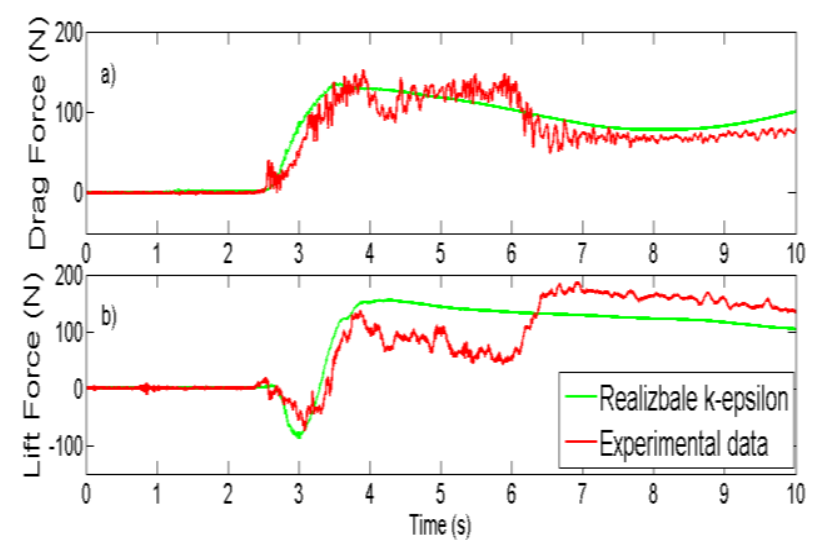

Figure 4 - Comparison between numerical model (Realizable k-epsilon turbulence model) and experimental data. Dry bed condition, $\mathrm{h}=50 \mathrm{~cm}, \mathrm{e} / \mathrm{D}=0.6$. a) Drag force time history, $R^{2}=0.8 \mathrm{~b}$ ) Lift force time history, $R^{2}=0.6$.

\section{CONCLUSIONS}

Evolution of the hydrodynamic forces exerted on a pipe due to dam break flow was studied experimentally and numerically. Based on the results of this study:

- $\quad$ For dry bed conditions, pipe elevation above the bed has a significant impact on both drag and lift force time history.

- For wet bed conditions, changing flow condition by changing still water depth to impoundment depth ratio $(\mathrm{d} / \mathrm{h})$ showed to significantly influence the force time history. However, changing pipe elevation above the bed did not have a considerable effect on the force behaviour.

- Among the different RANS turbulence models developed the realizable k-epsilon model showed the best agreement with the experimental results.

\section{REFERENCES}

ASCE/SEI (ASCE/structural Engineering Institute) (2017): Minimum design loads and associated criteria for buildings and other structures. ASCE/ SEI 7-16, Reston, VA, 25-50.

FEMA P646., 2012. Guidelines for design of structures for vertical evacuation from tsunamis. Federal Emergency Management Agency, Washington, D.C., 174 pp. 